\title{
Non-Zero Fermi Level Density of States for a Disordered d-Wave Superconductor in Two Dimensions
}

\author{
K. Ziegler ${ }^{a}$, M.H. Hettler ${ }^{b}$, and P.J. Hirschfeld ${ }^{b}$ \\ ${ }^{a}$ Max-Planck-Institut für Physik Komplexer Systeme, Außenstelle Stuttgart, Postfach 800665, D-70506 Stuttgart, Germany \\ ${ }^{b}$ Dept. of Physics, Univ. of Florida, Gainesville, FL 32611, USA.
}

(February 25, 2018)

\begin{abstract}
It has been known for some time that, in three dimensions, arbitrarily weak disorder in unconventional superconductors with line nodes gives rise to a nonzero residual density of zero- energy quasiparticle states $N(0)$, leading to characteristic low-temperature thermodynamic properties similar to those observed in cuprate and heavy-fermion systems. In a strictly two-dimensional model possibly appropriate for the cuprates, it has been argued that $N(0)$ vanishes, however. We perform exact calculations for $\mathrm{d}$ - and extended s-wave superconductors with Lorentzian disorder and similar models with continuous disorder distribution, and show that in these cases the residual density of states is nonzero even in two dimensions. We discuss the reasons for this discrepancy, and the implications of our result for the cuprates.
\end{abstract}

PACS numbers: 74.25-q, 74.25.Bt, 74.62.Dh

Introduction. A good deal of evidence [1] has accumulated recently suggesting that the order parameter in the cuprate superconductors vanishes linearly at lines on the Fermi surface. Frequently these experiments have been interpreted in terms of a $d_{x^{2}-y^{2}}$ pairing state, but states of "extended-s" symmetry with nodes have also been considered. One of the most interesting consequences of such nodes in three spatial dimensions is the creation of a nonzero density of zero-energy quasiparticle states $N(0)$ for infinitesimal disorder. [2,3] Such a residual density of states (DOS) is of course reflected in many experimental observables, and may be shown [4] to lead in particular to a $T^{2}$ term in the London penetration depth $\lambda(T)-\lambda(0)$, and more generally to low-temperature thermodynamic and transport properties characteristic of a normal Fermi system with strongly reduced DOS. Systematic $Z n$-doping and electron damage experiments have been found to lead to precisely these types of temperature dependences in $Y B C O$ single crystals [5], and in certain cases quantitative fits [6] have been obtained to the "dirty d-wave" model, in which the effects of potential scatterers on a $2 \mathrm{D}$ d-wave superconductor are calculated using a t-matrix approximation [7.8] assuming large electronic phase shifts.

Recently, Nersesyan et al. [9] have questioned the accuracy of the t-matrix approximation when applied to a strictly $2 \mathrm{D}$ disordered d-wave system, pointing out that in 2D logarithmic divergences in multisite scattering processes, some of which are neglected in the t-matrix approach, prevent a well-controlled expansion in impurity concentration. These authors avoid perturbation theory by using bosonization together with the replica trick, and predicted a power law DOS $N(E) \sim|E|^{\alpha}, \alpha \simeq 1 / 7$, for sufficiently small energy $E$ and disorder, rather than the analytic behavior $N(\omega) \sim$ const $+a E^{2}$ expected in $3 \mathrm{D}$. They also argued that a non-zero DOS at $E=0$, a quantity indicating spontaneous symmetry breaking, may not occur because of the Mermin-Wagner theorem [10].
Although the physical systems in question are in reality highly anisotropic 3D systems, the possibility of a 2D-3D crossover at low temperatures could conceivably invalidate some of the results of the usual "dirty d-wave" approach. This would render the description of the lowtemperature transport properties of the cuprate superconductors considerably more complicated even were the order parameter to correspond to the very simple $2 \mathrm{D}$ $d_{x^{2}-y^{2}}$ form usually assumed.

It is therefore of considerable importance to check the results of Ref. [9] by other methods. In this paper we show that for certain types of disorder, exact results can be obtained for the DOS of strictly 2D disordered superconductors. We show that for any disorder diagonal in position and particle-hole space, the DOS of a classic isotropic s-wave superconductor has a rigorous threshold at the (unrenormalized) gap edge $\Delta$, as expected from Anderson's theorem 11]. Within the same general method, we show that the residual DOS $N(0)$ of a superconductor with line nodes (e.g. d- or extended s-wave) is nonzero for arbitrarily small disorder, in disagreement with Ref. [9]. We believe that the DOS in a disordered system is not an order parameter which belongs to the class of order parameters covered by the Mermin-Wagner theorem. This is supported by the fact that a non-zero DOS occurs also in other tight-binding models (e.g., model for two-dimensional Anderson localization (12]), which are described by a field theory with continuous symmetry.

As exact results are only obtainable for Lorentzian disorder, we discuss ways 13 of obtaining information on the effects of other distributions, including models where the randomness has a compact domain. Finally, we compare our results to those arising from alternative methods, and comment on possible origins of the current disagreement. 
Density of states. Here, we introduce a general method of calculating exactly the DOS of a superconductor for certain types of disorder, motivated by the analysis of Dirac fermions in 2D. 14] The BCS Hamiltonian is given by

$$
H=\left(-\nabla^{2}-\mu\right) \sigma_{3}+\Delta \sigma_{1},
$$

which describes quasiparticles in the presence of the spin singlet order parameter $\Delta$. The $\sigma_{i}$ are the Pauli matrices in particle-hole space. The disorder is modeled by taking $\mu=\mu_{x}$ as a random variable distributed according to a probability distribution $P\left(\mu_{x}\right)$.

The kinetic energy operator $-\nabla^{2}$ is taken to act as $\nabla^{2} \Psi(x)=\Psi\left(x+2 e_{1}\right)+\Psi\left(x-2 e_{1}\right)+\Psi\left(x+2 e_{2}\right)+\Psi\left(x-2 e_{2}\right)$ on a function $\Psi(x)$ of the sites $x$ of a 2 D square lattice spanned by the unit vectors $e_{1}$ and $e_{2}$. Note this function involves displacements of two lattice sites rather than one, as would be the case in the simplest tightbinding representation of the lattice kinetic energy. For a system of fermions in the thermodynamic limit, the bare kinetic energy will then have a band representation quite similar to the usual tight-binding form, with no particular distinguishing features near the Fermi level. The reason for this choice will become clear below. It obeys, of course, the same global continuous symmetries discussed for the model in Ref. [9]. The bilocal lattice operator $\hat{\Delta} \equiv \Delta_{x, x^{\prime}}$ is taken to act as a cnumber in the isotropic s-wave case, $\hat{\Delta} \Psi(x)=\Delta \Psi(x)$, whereas to study extended pairing we define $\hat{\Delta}_{d}^{s} \Psi(x)=$ $\Delta_{d}^{s}\left[\Psi\left(x+e_{1}\right)+\Psi\left(x-e_{1}\right) \pm \Psi\left(x+e_{2}\right) \pm \Psi\left(x-e_{2}\right)\right]$.

We consider the single-particle Matsubara Green function defined as $G(i E)=\left(i E \sigma_{0}-H\right)^{-1}$. We are primarily interested in calculating the DOS $N(E) \equiv$ $-\frac{1}{\pi} \operatorname{Im} \sum_{\vec{k}}\left\langle G_{11}(\vec{k}, i E \rightarrow E+i \epsilon)\right\rangle$ where $\langle\ldots\rangle$ denotes the disorder average. The problem now is how to perform this disorder average over the probability measure $P\left(\mu_{x}\right) d \mu_{x}$ of the random variable $\mu_{x}$. Exact results for the disorder-averaged propagator in noninteracting systems can frequently be obtained for Lorentzian disorder, $P\left(\mu_{x}\right) d \mu_{x}=(\gamma / \pi)\left[\left(\mu_{x}-\mu_{0}\right)^{2}+\gamma^{2}\right]^{-1} d \mu_{x}$, by exploiting the simple pole structure of $P\left(\mu_{x}\right)$ in the complex $\mu_{x}$ plane. $\mu_{0}$ is the chemical potential of the averaged system. For convenience, we set $\mu_{0}=0$. The averaged Green function is $\langle G(i E)\rangle \equiv \int \prod_{x} d \mu_{x} P\left(\mu_{x}\right) G\left(i E ; \mu_{x}\right)$, which may then be trivially evaluated if $G$ can be shown to be analytic in either the upper or lower half-plane.

In a superconductor, the Green function depends on the random variable $\mu_{x}$ via $\mu_{x} \pm i E$, as a consequence of the particle-hole structure. Therefore, the averaging of $G$ with respect to Lorentzian disorder is not trivially possible. However, we will show below that it is possible to reformulate the problem so that $G$ consists of terms which are analytic in one of the half planes. This allows us to perform the averaging of the Green function for
Lorentzian disorder.

Isotropic s-wave superconductor. We first assume a homogeneous s-wave order parameter, neglecting the response of the superconducting condensate to the random potential. The Matsubara Green function may be written $G(i E)=-\left(i E \sigma_{0}+H\right)\left(E^{2}+H^{2}\right)^{-1}$ where we note that $H^{2}=\left(-\nabla^{2}-\mu\right)^{2} \sigma_{0}+\Delta^{2} \sigma_{0}$ since in the isotropic s-wave case, $\left(-\nabla^{2}-\mu\right) \sigma_{3}$ anticommutes with $\Delta \sigma_{1}$ even for random $\mu$ due to the locality of the order parameter.

The expression $H^{2}+E^{2}$ is proportional to the unit matrix; as a consequence, the Green function can be written in the simple form

$$
\begin{aligned}
G(i E)=-\frac{i E \sigma_{0}+H}{2 i \sqrt{\Delta^{2}+E^{2}}}[ & \left(-\nabla^{2}-\mu-i \sqrt{\Delta^{2}+E^{2}}\right)^{-1} \\
& \left.-\left(-\nabla^{2}-\mu+i \sqrt{\Delta^{2}+E^{2}}\right)^{-1}\right] \sigma_{0}
\end{aligned}
$$

It is straightforward to show that the imaginary part of this expression (after analytic continuation, $i E \rightarrow E+i \epsilon$ ) for any given configuration of impurities is vanishing for $|E|<\Delta$. Therefore, the DOS shows a gap of size $\Delta$ independent of the distribution function $P(\mu)$. Thus, our model reproduces the famous Anderson theorem 11. which states that the thermodynamics of an isotropic swave superconductor are not affected by diagonal, nonmagnetic disorder. The situation is different if the order parameter itself is random [13].

$d$ - and extended-s symmetry superconductors. The second class of examples includes the d-wave and extendeds "bond" order parameters $\hat{\Delta}_{d}^{s}$ defined above. The corresponding pure systems in momentum space fulfill the condition $\sum_{k} \Delta_{k}=0$, so that nonmagnetic disorder must cause significant pair breaking [2]. The behavior of the imaginary part of the Green's function can be studied using a method analogous to that used for the s-wave case. However, the main difference is that the nonlocal order parameter term $\hat{\Delta}_{d}^{s} \sigma_{1}$ does not anticommute with $\left(-\nabla^{2}-\mu\right) \sigma_{3}$ anymore if $\mu$ is random.

This requires a different type of transformation. We introduce a diagonal matrix (or staggered field) $D_{x, x^{\prime}}=$ $(-1)^{x_{1}+x_{2}} \delta_{x, x^{\prime}}$ (note $D^{2}$ is the unit matrix). Now we may write

$$
\begin{aligned}
H^{2}=H D \sigma_{3}^{2} D H= & {\left[\left(-\nabla^{2}-\mu\right) D \sigma_{0}-i \hat{\Delta}^{d}{ }^{s} D \sigma_{2}\right] } \\
& {\left[D\left(-\nabla^{2}-\mu\right) \sigma_{0}+i D \hat{\Delta}^{s}{ }^{s} \sigma_{2}\right] }
\end{aligned}
$$

Because $D$ commutes with $-\nabla^{2}$ (as defined above) and $\mu$, but anticommutes with the order parameter $\hat{\Delta}_{d}^{s}$, we have simply $H^{2}=\tilde{H}^{2}$, with $\tilde{H} \equiv\left(-\nabla^{2}-\mu\right) D \sigma_{0}-i \hat{\Delta}_{d}^{s} D \sigma_{2}$. Therefore, the quantity $H^{2}+E^{2} \sigma_{0}=\left(\tilde{H}+i E \sigma_{0}\right)(\tilde{H}-$ $\left.i E \sigma_{0}\right)$ can be used to write

$$
\begin{aligned}
G(i E)=\frac{i\left(i E \sigma_{0}+H\right)}{2 E} & \left(\left(\tilde{H}-i E \sigma_{0}\right)^{-1}\right. \\
& \left.-\left(\tilde{H}+i E \sigma_{0}\right)^{-1}\right)
\end{aligned}
$$


Observe that both $H$ and $\tilde{H}$ appear in this expression, but $H$ only in the numerator. Since $\left(\tilde{H} \pm i E \sigma_{0}\right)^{-1}$ is an analytic function of $\mu D$ in either the upper or lower $\mu D$ half plane and $P(\mu D)=P(\mu)$ we can now perform the disorder integration. The disorder averaged Matsubara Green function is translational invariant. Performing a spatial Fourier transform we replace $-\nabla^{2}$ by $\xi=\epsilon_{\vec{k}}-\mu_{0}$ and find

$$
\langle G(i E)\rangle=-\frac{(i E+i \gamma) \sigma_{0}+\xi \sigma_{3}+\hat{\Delta}_{d}^{s} \sigma_{1}}{(E+\gamma)^{2}+\xi^{2}+\left(\hat{\Delta}_{d}^{s}\right)^{2}} \equiv G(i E+i \gamma) .
$$

This is the Matsubara Green function of the pure system with the frequency $i E$ shifted by the disorder parameter, $i E \rightarrow i E+i \gamma$. It should be noted that for the local (isotropic) s-wave order parameter discussed before the average over a Lorentzian distribution in Eq. 2 implies a shift $i \sqrt{\Delta^{2}+E^{2}} \rightarrow i \sqrt{\Delta^{2}+E^{2}}+i \gamma$.

To obtain the DOS for the d-wave case we approximate the sum over the momenta $\vec{k}$ in standard fashion as $N_{o} \int_{-\infty}^{\infty} d \xi \int_{0}^{2 \pi} \frac{d \phi}{2 \pi}$ where $N_{o}$ is the density of states of the normal metal at the Fermi level, with the tetragonal Fermi surface approximated by a circle. The result is

$$
N(E)=N_{0} \int_{0}^{2 \pi} \frac{d \phi}{2 \pi} \operatorname{Im}\left(\frac{E+i \gamma}{\left(\Delta_{d}^{2}(\phi)-(E+i \gamma)^{2}\right)^{1 / 2}}\right)
$$

where the d-wave order parameter is approximated by $\Delta_{d}(\phi)=\Delta_{d} \cos (2 \phi)$. At $E=0, N(0)=$ $N_{0} \frac{2 \gamma}{\pi \Delta_{d}} \ln \left(4 \Delta_{d} / \gamma\right)$ for $\gamma<<\Delta_{d}$. Thus, the DOS is nonzero at the Fermi level for arbitrarily small disorder. For small $E, N(E)$ varies as $E^{2}$.

For more general continuous distributions $P(\mu) d \mu$ the averaged DOS can be estimated using again the analytic structure of $\tilde{G}$. Applying the ideas of Ref. [13], one can derive a lower bound by a decomposition of the lattice into finite sub-squares. The average DOS on an isolated sub-squares can be estimated easily. Moreover, the contribution of the connection between the subsquares to the average DOS can also be estimated. A combination of both contributions leads to $\langle N(0)\rangle \geq$ $c_{1} \min _{-\mu_{1} \leq \mu \leq \mu_{1}} P(\mu)$, where $c_{1}$ and $\mu_{1}$ are distribution dependent positive constants. In particular, $\mu_{1}$ must be chosen such that the spectrum of $H\left(\mu_{0}=0\right)=$ $-\nabla^{2} \sigma_{3}-i \hat{\Delta}_{d}^{s} D \sigma_{1}$ is inside the interval $\left[-\mu_{1}, \mu_{1}\right]$. For all unbounded distributions, like the Gaussian distribution used in Ref. [9], as well as compact distributions with sufficiently large support this estimate leads to a nonzero DOS at the Fermi level.

Discussion and comparison to other methods. The major result in the $\mathrm{d}$-wave (extended $\mathrm{s}$-wave) case with Lorentzian disorder is the presence of a finite purely imaginary self energy $\Sigma_{0}=-i \gamma \sigma_{0}$ due to nonmagnetic disorder which leads to a nonzero DOS at the Fermi level.
The latter is in qualitative agreement with standard theories based on the self-consistent t-matrix approximation [7,8] as well as with exact diagonalization studies in 2D 16]. In contrast to such theories our self energy has no dependence on $\hat{\Delta}_{d}^{s}$, i.e. it is the same as in the normal state. In Fig. 1 we show a comparison of the self energies of our theory and the limits of the t-matrix approximation.

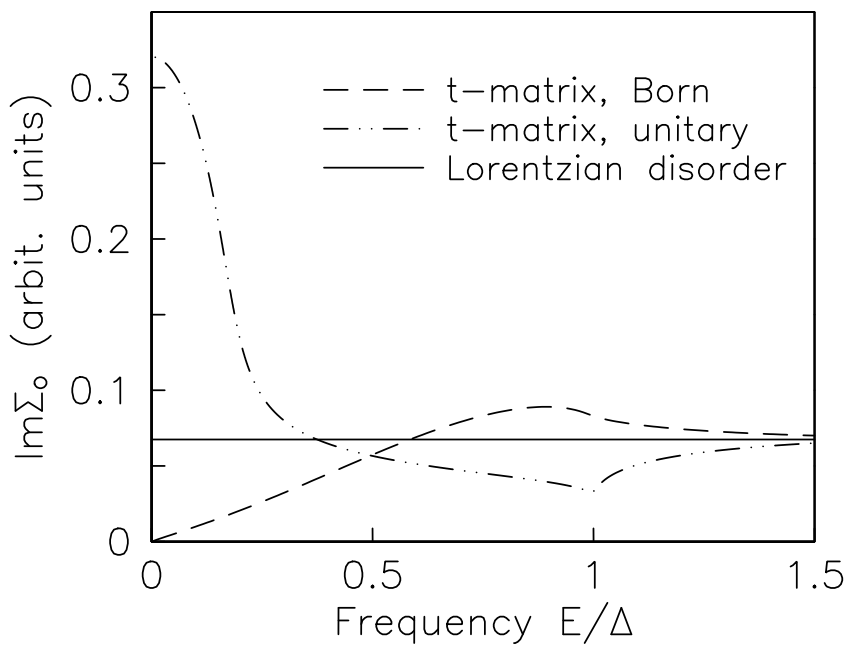

FIG. 1. Imaginary part of the self energy vs. frequency. For Lorentzian disorder (solid line) the self energy is constant $i \gamma$. The self energy of the self-consistent $\mathrm{t}-$ matrix approximation in the unitary scattering limit (dashed-dotted line) behaves $\propto(\delta \Delta)^{1 / 2}$ at zero frequency. For Born scattering (dashed line) the value at zero frequency is nonzero, but exponentially small. We have adjusted the impurity concentration to obtain equal normal state self energies for the $t$-matrix results.

A drawback of the model with Lorentzian disorder is that impurity concentration does not appear explicitly in the theory. Whereas in the t-matrix approach we have with the impurity concentration and the scattering strength (or phase shift) two parameters associated with disorder, in the present model we have only $\gamma$, the width of the Lorentzian. A way of making a connection is by comparing the variance of the Lorentzian distribution $(\gamma)$ and the variance of the distribution underlying the $t-$ matrix approximation, which is a bimodal distribution of a chemical potential $\mu=\mu_{0}$ with probability $1-\delta$ ( $\delta$ being the dimensionless impurity concentration) and $\mu=\mu_{0}+V$ with probability $\delta$ ( $V$ being the scattering potential). The variance $\operatorname{Var}_{\mu}$ of this distribution is determined by

$$
\operatorname{Var}_{\mu}^{2}=\left\langle\mu^{2}\right\rangle-\langle\mu\rangle^{2}=V^{2}\left(\delta-\delta^{2}\right) .
$$

For small concentrations of impurities, $\delta<<1$, we find $\operatorname{Var}_{\mu}=V \delta^{1 / 2}$. The $\delta^{1 / 2}$ behavior is also found for 
$\operatorname{Im} \Sigma_{o}(E=0)$ in the t-matrix approach for strong scattering. Since in our model the variance of the distribution is also the imaginary part of the self energy, this suggests that our model is closer to the strong scattering limit of the $\mathrm{t}-$ matrix approximation than the Born limit.

Finally, we comment on the discrepancies between our result and the calculation of Nersesyan et al., who found a power law for the averaged DOS with Gaussian disorder. One might question the analysis of Nersesyan et al. because of the use of the replica trick, which is a dangerous procedure in a number of models. [17] However, Mudry et al. 18 have obtained identical results for the continuum problem of Dirac fermions in the presence of a random gauge field using supersymmetry methods. We therefore believe that the crucial difference between our results and those of Ref. [9] occurs in the passage to the continuum and concomitant mapping of the site disorder in the original problem onto the random gauge field. Only in the continuum case is there a direct analogy between disorder in the chemical potential and a gauge field; on the lattice, gauge fields and chemical potential terms enter quite differently. First, chemical potential terms are local while gauge fields are defined on bonds. Furthermore, chemical potential disorder enters linearly in the Hamiltonian while gauge fields enter through the Peierls prescription as a phase in the exponential multiplying the kinetic energy.

Disorder of the gauge field type is furthermore nongeneric even in the continuum, as discussed by Mudry et al., who showed that the critical points of the system with random gauge field are unstable with respect to small perturbations by other types of disorder. [20] We expect that a proper mapping of the lattice Dirac fermion or $\mathrm{d}$ wave superconductor problems to continuum models will inevitably generate disorder other than random gauge fields. Therefore, we believe that our result of a finite DOS at the Fermi level is the generic case for a d-wave superconductor in two dimensions.

Conclusions. We have computed the single particle Green function and DOS for a model of a superconductor with nonmagnetic impurities. For an isotropic s-wave superconductor, we recover standard results; in particular, Anderson's theorem is reproduced. Our calculations for the disorder-averaged $\mathrm{d}$ - and extended s-wave propagators show the DOS is nonzero for all energies, provided the distribution of the chemical potential is continuous and of sufficient width. The disorder average has been performed exactly in the case of a Lorentzian distribution. Our calculation casts doubt on the result by Nersesyan et al., who found a power law for the averaged DOS with Gaussian disorder, and suggests that the standard $\mathrm{t}$-matrix approach to disordered d-wave superconductors is qualitatively sufficient.

Acknowledgements. The authors gratefully acknowledge discussions with R. Fehrenbacher and C. Mudry. They are also grateful for the support of the Institute for Fundamental Theory, University of Florida, and the Institut für Theorie der Kondensierten Materie, Universität Karlsruhe.

[1] For recent reviews see J. Annett, N. Goldenfeld, and A. Leggett, LANL cond-mat/9601060 and D.J. Scalapino, Physics Reports 250, 329 (1995).

[2] L.P. Gor'kov and P.A. Kalugin, Pis'ma Zh. Eksp. Teor. Fiz. 41, 208 (1985) [JETP Lett. 41, 253 (1985)].

[3] K. Ueda and T. M. Rice in "Theory of Heavy Fermions and Valence Fluctuations", eds. T. Kasuya and T. Saso, Springer Series in Solid State Sciences, vol. 62, p. 267 (1985).

[4] F. Gross et al., Z. Phys. B 64, 175 (1986).

[5] Y. Kitaoka et al., J. Phys. Soc. Japan 63, 2052 (1994); D. A. Bonn et al., Phys. Rev. B 50, 4051 (1994); J. Giapintzakis et al., Phys. Rev. B ; preprint 1995.

[6] P.J. Hirschfeld, W.O. Putikka, and D.J. Scalapino, Phys. Rev. B 50, 10250 (1994).

[7] P. J. Hirschfeld et al., Sol. St. Commun. 59, 111 (1986).

[8] S. Schmitt-Rink et al., Phys. Rev. Lett. 57, 2575 (1986).

[9] A. A. Nersesyan, A. M. Tsvelik and F. Wenger, Phys. Rev. Lett. 72, 2628 (1994); Nucl. Phys. B 438, 561 (1995).

[10] N. D. Mermin and H. Wagner, Phys. Rev. Lett. 17, 1133 (1966).

[11] P. W. Anderson, J. Phys. Chem. Solids 11, 26 (1959).

[12] P. A. Lee and T. V. Ramakrishnan, Rev. Mod. Phys. 57, 287 (1985).

[13] K. Ziegler, Comm. Math. Phys. 120, 177 (1988).

[14] K. Ziegler, Phys. Rev. B 53, 9653 (1996).

[15] Y. Hatsugai and P.A. Lee, Phys. Rev. B 48, 4204 (1993).

[16] T. Xiang and J.M. Wheatley, Phys. Rev. B 51, 11721 (1995).

[17] K. Ziegler, Phys. Rev. Lett. 73, 3488 (1994).

[18] C. Mudry, C. Chamon and X.-G. Wen, preprint, condmat/9509054

[19] J.-S. Caux, I. I. Kogan and A. M. Tsvelik, preprint, hepth/9511134

[20] It should be mentioned that even in the case of a random gauge field it is not established [18,19] that the DOS indeed vanishes at the Fermi level. 